\title{
Coupling of upper and lower limb pattern generators during human crawling at different arm/leg speed combinations
}

\author{
M. J. MacLellan • Y. P. Ivanenko · G. Catavitello • \\ V. La Scaleia $\cdot$ F. Lacquaniti
}

Received: 23 October 2012 / Accepted: 26 November 2012 / Published online: 16 December 2012

(C) Springer-Verlag Berlin Heidelberg 2012

\begin{abstract}
A crawling paradigm was performed by healthy adults to examine inter-limb coupling patterns and to understand how central pattern generators (CPGs) for the upper and lower limbs are coordinated. Ten participants performed hands-and-feet crawling on two separate treadmills, one for the upper limbs and another one for the lower limbs, the speed of each of them being changed independently. A 1:1 frequency relationship was often maintained even when the treadmill speed was not matched between the upper and lower limbs. However, relative stance durations in the upper limbs were only affected by changes of the upper limb treadmill speed, suggesting that although absolute times are adjusted, the relative proportions of stances and swing do not adapt to changes in lower limb treadmill speeds. With large differences between treadmill speeds, changes in upper and lower limb coupling ratio tended to occur when the upper limbs stepped at slower speeds than the lower limbs, but more rarely the other way around. These findings are in sharp contrast with those in the cat, where forelimbs always follow the rhythm of the faster moving hindlimbs. However, the fact that an integer frequency ratio is often maintained between the upper and lower limbs supports evidence of coupled CPG control. We speculate that the preference for
\end{abstract}

M. J. MacLellan $(\bowtie) \cdot$ Y. P. Ivanenko · G. Catavitello ·

V. La Scaleia $\cdot$ F. Lacquaniti

Laboratory of Neuromotor Physiology, IRCCS Fondazione

Santa Lucia, via Ardeatina 306, 00179 Rome, Italy

e-mail:m.maclellan@hsantalucia.it

F. Lacquaniti

Centre of Space Bio-medicine, University of Rome

Tor Vergata, 00173 Rome, Italy

F. Lacquaniti

Department of Systems Medicine, University

of Rome Tor Vergata, 00133 Rome, Italy the upper limb to decrease step frequency at lower speeds in humans may be due to weaker ascending propriospinal connections and/or a larger influence of cortical control on the upper limbs which allows for an overriding of spinal CPG control.

Keywords Quadrupedal locomotion·Arm/leg coordination $\cdot$ Spinal cord $\cdot$ Central pattern generator

\section{Introduction}

The motion of upper and lower limbs during upright walking at natural and faster speeds tends to be tightly coupled in a 1:1 frequency relationship, even though the arms are not essential to walking in humans (Webb et al. 1994; Donker et al. 2002; Dietz and Michel 2009). The same seems to be true also for unperturbed hand-and-foot crawling in healthy (Sparrow and Newell 1994; Getchell et al. 2001; Patrick et al. 2009) and pathological (Tan 2010) adults. This coupling presumably reflects both biomechanical and neural linkages between cervical and lumbosacral central patterns generators (CPGs) that control the upper and lower limbs, respectively (Dietz et al. 2001; Ivanenko et al. 2005; Zehr et al. 2009). These CPGs have been documented more thoroughly in quadrupedal mammals (Orlovsky et al. 1999; Falgairolle et al. 2006).

However, motion of the upper and lower limbs is not always coupled in a 1:1 frequency relationship: for instance, a $2: 1 \mathrm{arm} / \mathrm{leg}$ frequency ratio is observed during human bipedal walking at low speeds (Webb et al. 1994). In addition, Wannier et al. (2001) observed that the frequency relationship between the upper and lower limbs was not 1:1 during a swimming task in humans (though it was consistently an integer ratio). Such frequency relationships can be 
investigated during quadrupedal locomotion in paradigms in which the fore and hindlimbs walk on separate treadmills. In the decerebrate cat, slowing of the hindlimbs leads to a change in frequency relationship (2:1 fore/hindlimb ratio), while a 1:1 frequency relationship was maintained when the forelimb speed was lowered (Akay et al. 2006). This difference between limbs was attributed to asymmetric ascending and descending propriospinal pathways. Human crawling shares a number of common features with animal quadrupedal locomotion (de Sèze et al. 2008; Patrick et al. 2009; MacLellan et al. 2012b). However, it remains unclear whether or not the coupling mode between the upper and lower limbs is similar to that of other quadrupeds.

In the current study, we used the crawling paradigm in adult humans to 1) determine which step characteristics are adjusted by the central nervous system (CNS) in order to maintain a 1:1 frequency relationship when treadmill speeds for the upper and lower limbs are manipulated independently and 2) examine how the relationship between the upper and lower limbs changes when the limbs no longer follow a 1:1 frequency ratio. It is hypothesized that a 1:1 frequency relationship is maintained through fine adjustments of a cycle period as well as stance and swing times in both the upper and lower limbs. In addition, it is hypothesized that an integer relationship will be maintained when the upper and lower limbs deviate from a 1:1 frequency ratio, as seen previously during swimming (Wannier et al. 2001).

\section{Methods}

\section{Participants}

Ten healthy adults ( 3 females $/ 7$ males, age $32.0 \pm 9.3$ years, mass $73.4 \pm 11.2 \mathrm{~kg}$ ) participated in the study. The studies were in accordance with the Declaration of Helsinki, and informed consent was obtained from all the participants according to the procedures approved by the Ethics Committee at the Santa Lucia Foundation.

\section{Protocols}

Participants performed hands-and-feet crawling on 2 separate treadmills such that the hands and feet moved on separate treadmills (Fig. 1a). The experiment was separated into 2 blocks. In the first block, upper and lower limb treadmill speeds were manipulated $(1,2$, and $3 \mathrm{~km} / \mathrm{h}$ each), resulting in 9 treadmill speed conditions which were presented randomly. In the second block of conditions, extreme treadmill differences were presented (upper limbs: $0.5 \mathrm{~km} / \mathrm{h}$, lower limbs 3 or $4 \mathrm{~km} / \mathrm{h}$ and vice versa) to encourage limb frequency relationships other than $1: 1$. For each speed condition, participants were provided with a short adaptation a

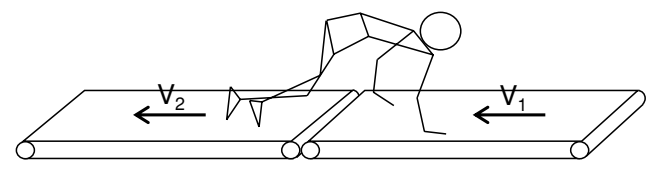

b

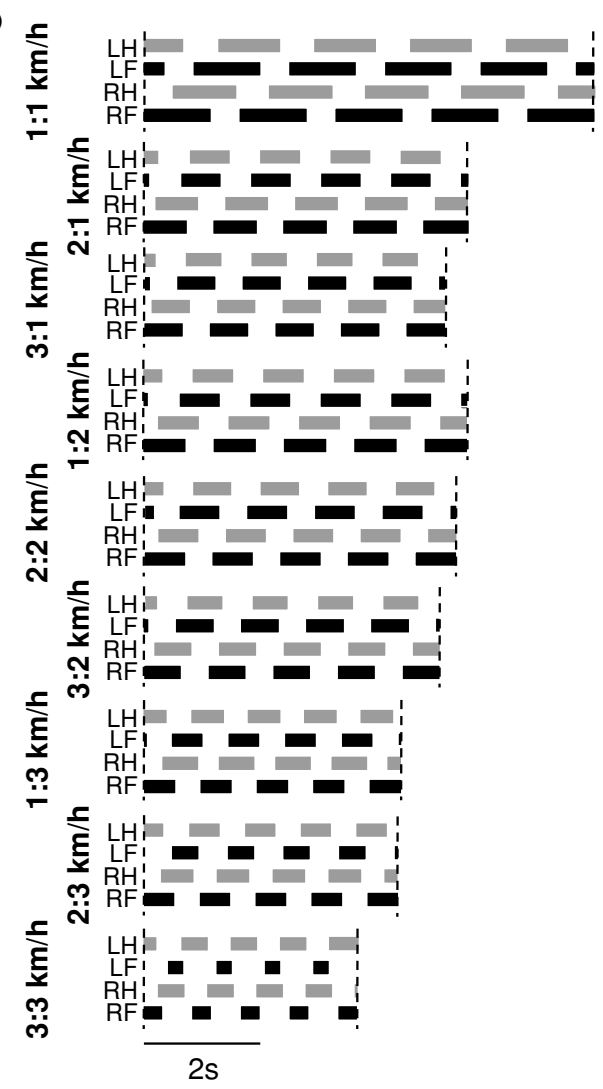

Fig. 1 Experimental setup (a) and limb contact patterns (b) from a sample participant across different speed (arm/leg) combinations $(L H$ left hand, $L F$ left foot, $R H$ right hand, $R F$ right foot). The top panel (a) illustrates hand-and-foot crawling on treadmills moving at separate speeds $\left(\mathrm{V}_{1}\right.$ and $\left.\mathrm{V}_{2}\right)$. Limb contact patterns in (b) are indicated by solid bars (stance). Only 1:1 frequency relationship cases are illustrated here

period (approximately 5-15 s), after which a minimum of 15 strides were performed ( 10 strides were used in the data analysis). Rest periods $(\sim 2 \mathrm{~min})$ were provided between conditions in order to avoid fatigue. The total duration of the experiments was about $1.5-2 \mathrm{~h}$.

\section{Data recording}

Full body 3-dimensional kinematic data were collected with a 9-camera Vicon-612 system (Oxford, UK) sampled at $100 \mathrm{~Hz}$. Reflective markers (diameter $1.4 \mathrm{~cm}$ ) were placed on $\mathrm{C} 7$ and L2 as well as bilaterally on the acromioclavicular joint, lateral epicondyle of the humerus, distal head of the ulna, end of 3rd distal phalanx, greater trochanter, lateral femoral condyle, lateral malleolus, calcaneus, head of 5th 
metatarsal, and end of 1 st metatarsal. All kinematic data were filtered using a 2nd order dual-pass Butterworth filter with a low-pass cutoff frequency of $7 \mathrm{~Hz}$.

Data analysis

From the kinematic data, limb contacts and liftoffs were computed from the minimum vertical velocity peaks (when marker velocity decreased past a threshold of $0.05 \mathrm{~m} / \mathrm{s}$ ) from the wrist and end of 1st metatarsal for the upper and lower limbs, respectively. From these values, cycle periods were calculated from the time between 2 contacts in each limb. The stance to swing transitions were calculated using the initiation of elevation of the end of 3rd distal phalanx and end of 1st metatarsal for the upper and lower limbs, respectively (when vertical marker velocity increased past a threshold of $0.05 \mathrm{~m} / \mathrm{s}$ ). The lower and upper limb excursions were defined as the distance between two successive foot and arm touchdown events of the right limb.

The relationship between arm and leg cycle frequency under various conditions was also investigated. The frequency of limb motion was determined by applying a Fourier transform (Matlab function "fft") to the anterior/posterior trajectories of the wrist and the end of 1st metatarsal (after subtraction of the position of the shoulder and greater trochanter to account for full body movement on the treadmill) for the upper and lower limbs, respectively. The peak harmonic from the Fourier spectrum was used to estimate limb motion frequency for the lower and upper limbs (Wannier et al. 2001), and the ratio between limb frequencies was determined. Frequency ratios are consistently stated as upper/lower limb relationships.

In addition, the coordination between the upper and lower limbs was determined using ipsilateral phase lag (IPL), in which the timing of the upper limb contact $(\mathrm{t})$ is represented by a percentage between lower limb contacts (Patrick et al. 2009; MacLellan et al. 2012b):

$\mathrm{IPL}=\frac{t}{T} \times 100 \%$,

where $T$ is lower limb cycle duration. This measure was used for two reasons in the current study. The first reason was to determine if individual limb coordination patterns were related to displaying limb coupling ratios other than 1:1. During quadrupedal locomotion, lateral gait patterns (ipsilateral upper/lower limb contact at similar instances) are determined at a value of $0 \%$, and diagonal gait patterns (contralateral upper/lower limb contact at similar instances) are determined at a value of $50 \%$. The second reason was to more closely examine limb placement patterns in a small number of trials in which integer limb coupling ratios were not present. In these cases, the IPL parameter was calculated more than 1 time during the gait cycle. It should be noted that the IPL parameter is not generally used in such cases and has no physiological meaning for this specific use.

The results of this study (110 trials total) were separated into two parts. In the first section (90 trials), only trials with treadmill speeds of 1,2 , and $3 \mathrm{~km} / \mathrm{h}$ with participants crawling at a 1:1 frequency ratio between the upper and lower limbs were retained for the analysis. To determine this, the upper limb frequency was divided by the lower limb frequency, and trials were discarded (6 trials out of 90) if this value was not between 0.9 and 1.1. In the second part of the analysis, all trials are presented (110 trials).

\section{Statistics}

Descriptive statistics included the calculation of the mean and standard deviation (SD). Statistical analysis was performed using an upper limb $(1,2$, and $3 \mathrm{~km} / \mathrm{h})$ by lower $\operatorname{limb}(1,2$ and $3 \mathrm{~km} / \mathrm{h})$ treadmill speed two-way ANOVA for cycle period, stance and swing times, independently for the upper and lower limb movement. Reported results are considered significant for $p<0.05$.

\section{Results}

Tied treadmill speed conditions

When crawling on treadmills moving at the same speed, expected spatiotemporal patterns for the upper and lower limbs were found (Fig. 2, dashed lines). For example, the cycle period and the percentage duration of stance relative to the gait cycle decreased as treadmill speed increased. In addition, the excursion of the limbs remained relatively constant in each speed condition. Such patterns have been expanded upon in previous work (MacLellan et al. 2012b).

Limbs coupled in 1:1 frequency relationship: treadmills moving at differing speeds

Throughout most treadmill speed combinations, participants were able to maintain a 1:1 frequency relationship between the upper and lower limbs. Figure $1 b$ illustrates sample limb contact patterns from a representative participant over treadmill conditions ranging from 1 to $3 \mathrm{~km} / \mathrm{h}$. Upper and lower limb stride characteristics are illustrated in Fig. 2, and the results are presented below.

As expected, limb cycle periods decreased with faster treadmill belt speeds (Fig. 2a). Lower limb cycle periods were affected by changes in both the upper and lower limb treadmill speeds. However, cycle period was relatively greater when both treadmills were moving at $1 \mathrm{~km} / \mathrm{h}$, leading to an interaction effect $\left(F_{(4,71)}=10.36, p<0.001\right)$. In the upper limbs, a similar interaction effect was shown 


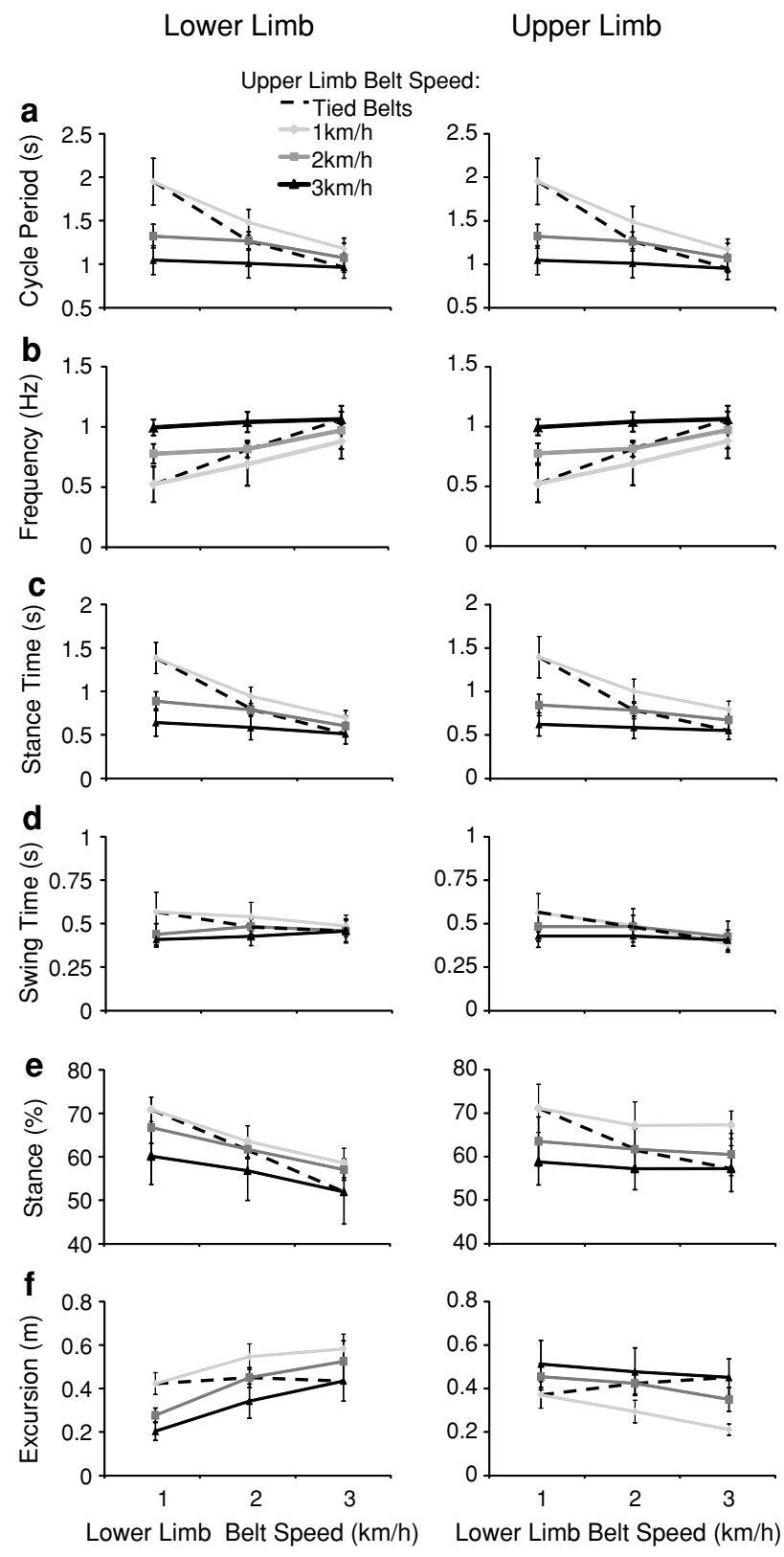

Fig. 2 Gait parameters when crawling was coupled with a 1:1 frequency relationship between the arm and leg motion (trials with differing ratios were discarded). Leg treadmill speeds are indicated on the $x$-axis, and arm treadmill speeds are represented by differing lines. Dashed lines represent tied treadmill speed conditions. Mean \pm SD is indicated for each treadmill speed combination

$\left(F_{(4,71)}=10.13, p<0.001\right)$. Fourier transforms of limb trajectories (Fig. 2b) suggested that fundamental harmonics increased with higher treadmill speeds, although interaction effects for the lower $\left(F_{(4,71)}=3.13, p<0.020\right)$ and upper $\left(F_{(4,71)}=2.80, p<0.032\right)$ limbs were shown due to low frequencies when both treadmills were moving at $1 \mathrm{~km} / \mathrm{h}$. These similarities in results are expected since the frequency ratio between the upper and lower limbs was 1:1.
The stance time patterns between treadmill speed combinations were similar to those of cycle duration (Fig. 2c). Interaction effects for the stance time of lower $\left(F_{(4,71)}=11.90, p<0.001\right)$ and upper $\left(F_{(4,71)}=9.19\right.$, $p<0.001)$ limbs suggested decreases in stance time with increasing treadmill speeds (although greater changes were shown for the $1: 1 \mathrm{~km} / \mathrm{h}$ condition, leading to the interaction effect). Swing time patterns between the upper and lower limbs were quite different than that of stance time, but the changes in swing time were minimal compared to those of stance time (Fig. 2d). Swing time in the lower limbs was only affected by changes in upper limb treadmill speed (main effect: $F_{(2,71)}=13.29, p<0.001$ ), while in the upper limbs, swing times were adjusted for changes in both upper $\left(F_{(2,71)}=3.73, p<0.029\right)$ and lower $\left(F_{(2,71)}=8.16\right.$, $p<0.001)$ treadmill belt speeds. Examination of stance proportions (Fig. 2e) in the lower limbs showed decreases as the speed of the upper $\left(F_{(2,79)}=19.40, p<0.001\right)$ and lower $\left(F_{(2,79)}=28.90, p<0.001\right)$ limb treadmill speeds increased. However, only changes in upper limb treadmill speeds resulted in changes in upper limb stance proportion $\left(F_{(2,79)}=28.06, p<0.001\right)$.

In addition, increasing treadmill speeds had opposite effects on excursion in the upper and lower limbs (Fig. 2f). The excursions of the lower limbs increased with lower limb treadmill speeds $\left(F_{(2,71)}=70.46, p<0.001\right)$ and decreased with greater upper limb treadmill speeds $\left(F_{(2,71)}=51.69\right.$, $p<0.001)$. In contrast, the excursions of the upper limbs decreased with greater lower limb treadmill speeds $\left(F_{(2,71)}=13.74, p<0.001\right)$ and increased with upper limb treadmill speeds $\left(F_{(2,71)}=41.10, p<0.001\right)$.

\section{Deviations from 1:1 coupling frequency ratio}

During treadmill speed conditions in which there was a large difference between the upper and lower limbs (i.e., upper limbs at $0.5 \mathrm{~km} / \mathrm{h}$ and lower limbs at $3 \mathrm{~km} / \mathrm{h}$ or vice versa), the majority of participants tended to deviate from a 1:1 frequency relationship. Figure 3 a shows limb contact patterns in participants who displayed limb frequency relationships of the legs cycling 2 and 3 times for each arm cycle, as well as 2 arm cycles for each leg cycle. When looking at the numbers of participants who displayed each type of coupling pattern, we found that a greater number of participants deviated from a 1:1 coupling ratio when the upper limb treadmill speed was lowered when compared to the lower limb (Fig. 3b).

To investigate frequency relationships between the upper and lower limb motion, a Fourier transform was used. Figure 4a illustrates upper and lower limb motion in a representative participant and the power spectrum associated with these trajectories. When all coupling ratios are plotted, limb motion generally cycled at integer frequency ratios 
a

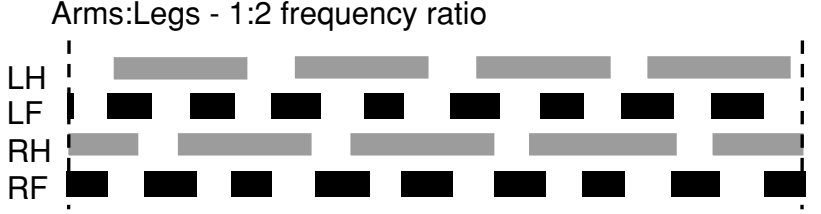

Arms:Legs - 1:3

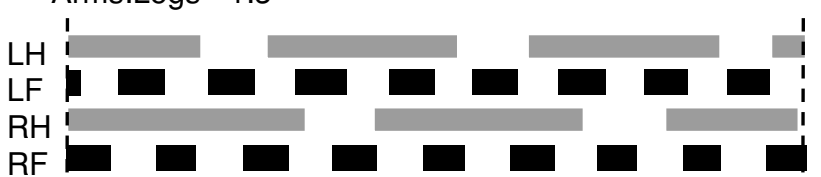

Arms:Legs - 2:1

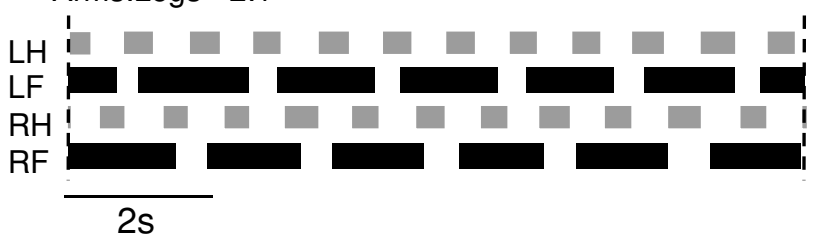

b
Proportion of participants who decreased arm frequency (\#)

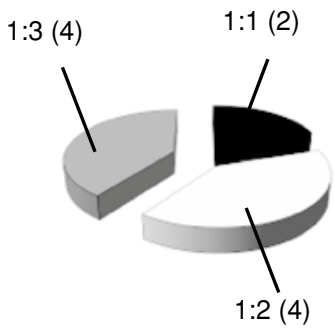

2:1 (3)

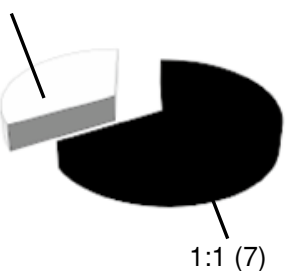

Fig. 3 Representative limb contact patterns from different participants (a) and proportions of participants displaying different coupling patterns (b) when a 1:1 frequency relationship was no longer maintained. As in Fig. 1, stance phases are indicated by a solid bar (a). The numbers in the parentheses in $\mathbf{b}$ indicate the number of subjects (out of a total of 10) that showed this pattern during the treadmill speed conditions

(Fig. 4b). Frequency ratios of 1:2 and 1:3 were observed when upper limb treadmill speed was decreased, while only frequencies of 2:1 were observed at decreased lower limb treadmill speeds.

It is known that human subjects show variable styles of crawling from diagonal to lateral gait (Patrick et al. 2009; MacLellan et al. 2012b). In the current study, we did not find a systematic relationship between the style of tied-belt crawling (diagonal versus lateral) and a preference for upper or lower limbs to cycle at lower frequencies. For instance, decreased upper limb frequencies were observed in participants who displayed both diagonal (Fig. 5a) and lateral (Fig. 5b) crawling styles. This technique also allowed us to further examine participants who did not display an integer frequency relationship between upper and lower limbs. In such instances, one of 2 patterns was observed. We observed a frequency drift
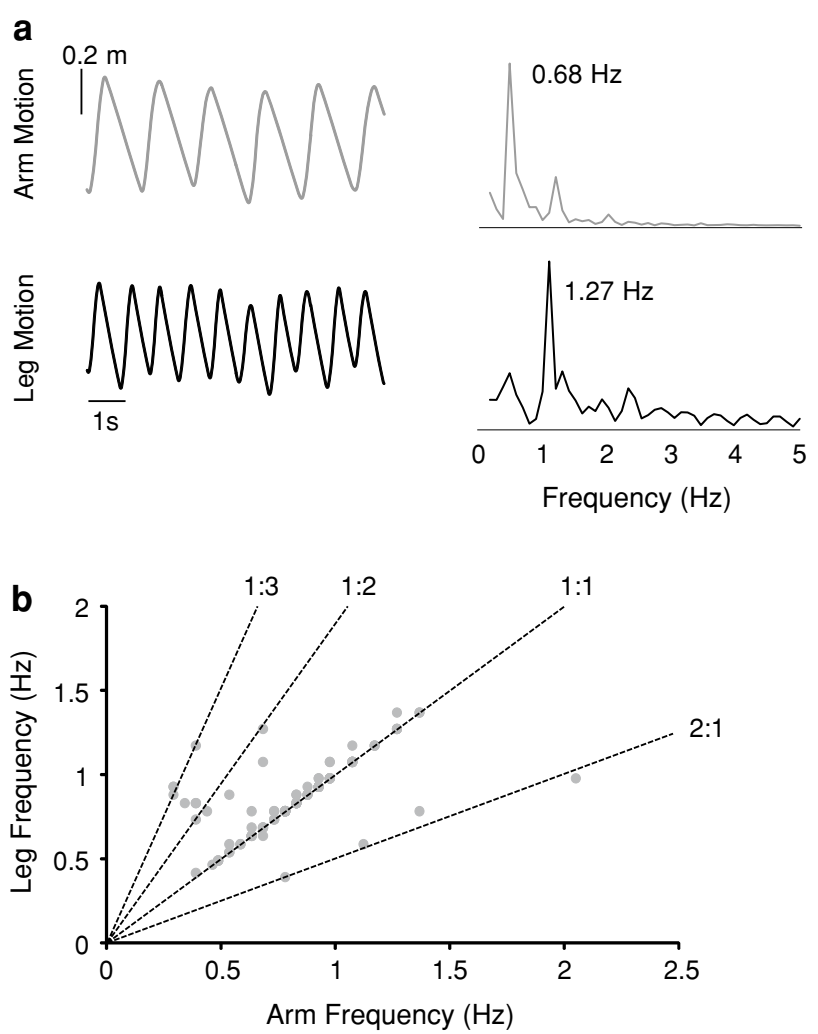

Fig. 4 Sample Fourier transform from a participant crawling at a 1:2 relationship (a) and group results illustrating limb frequency ratios (b). The anterior-posterior motions of the ulnar process and 1st metatarsal markers were used to determine arm and leg motion, respectively (upward and downward directions represent movement in the forward and backward directions, respectively). Numbers on the right side of a denote the peak frequency in the obtained spectrum. In b, each point illustrates the arm or leg movement frequency over the entire trial for a single participant and the dotted lines indicate integer arm/leg frequency ratios

in 3 cases, (Fig. 5b) and a phase resetting in 4 cases (Fig. 5c). This shows that even though these participants did not display a true integer frequency ratio, it appears in these trials participants were actually searching for a rhythm that was suitable for the current treadmill speed combination.

\section{Discussion}

The purpose of this study was twofold: to determine which step characteristics are adjusted by the central nervous system (CNS) in order to maintain a 1:1 frequency relationship when the upper and lower limbs are crawling at different speeds and examine how the relationship between the upper and lower limbs changes when the limbs no longer follow a 1:1 frequency ratio. As expected, a 1:1 frequency relationship was achieved by adjusting cycle period as well as stance and swing times, confirming our first hypothesis. However, 


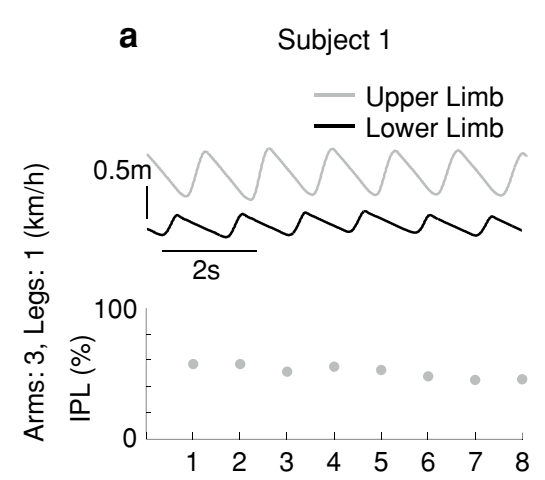

b Subject 2

C

Subject 3
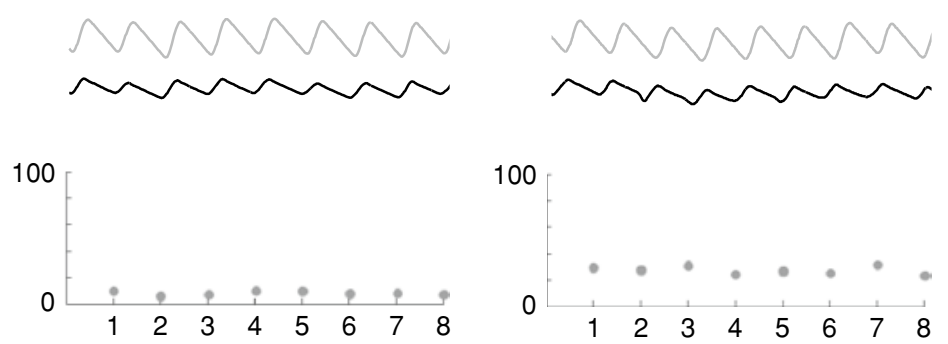

100
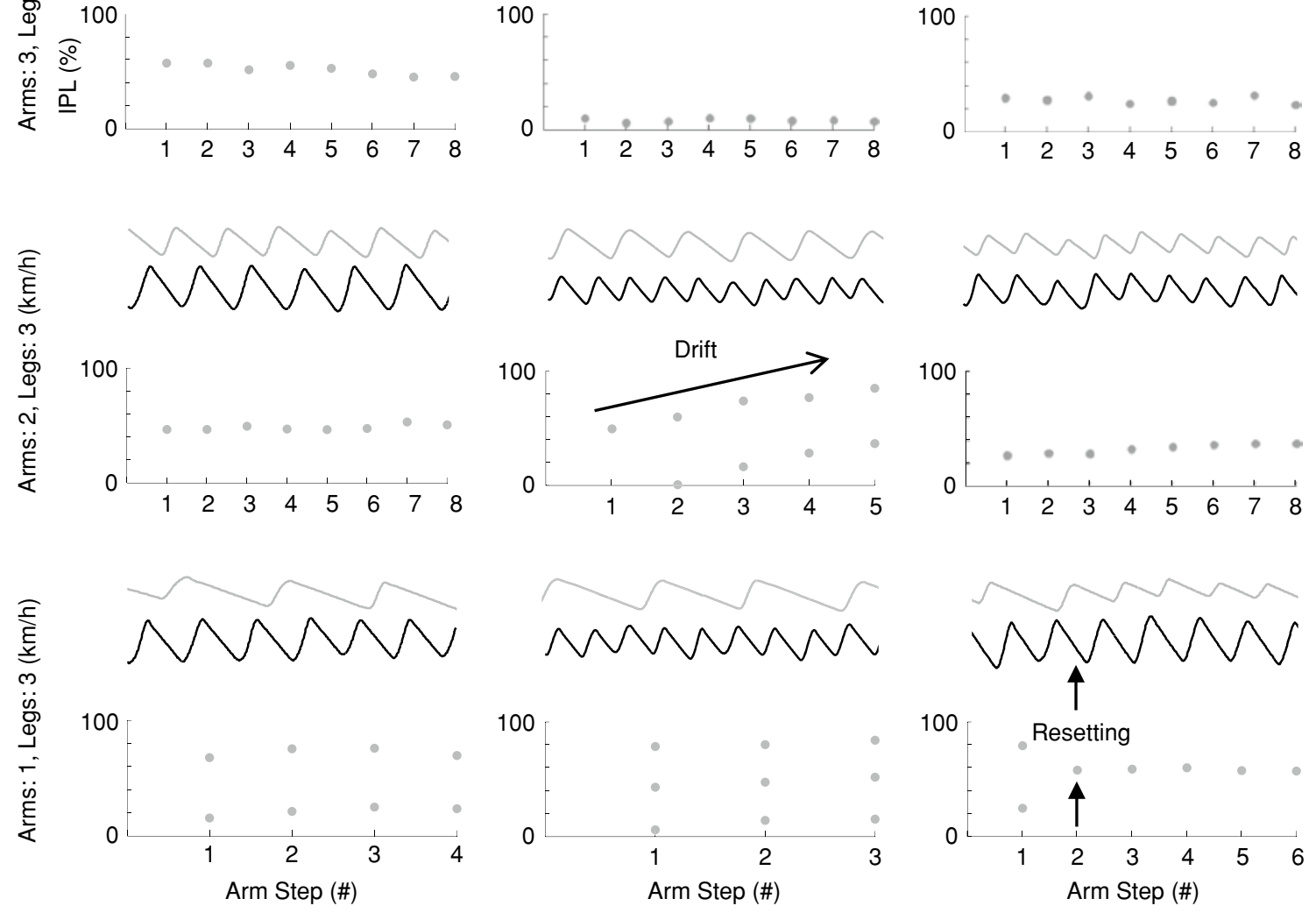

Fig. 5 Examples of inter-limb coordination patterns for different participants. The participant in a consistently displays integer (1:1 and 1:2) frequency ratios for all conditions. b Illustrates a participant who displays a frequency drift occurs for the $2: 3 \mathrm{~km} / \mathrm{h}$ condition. The

upper limb stance proportions were only affected by manipulations of upper limb treadmill speed (Fig. 2). In most of the treadmill speed combinations, an integer frequency ratio was maintained between the upper and lower limbs even when a 1:1 relationship was not present, therefore confirming our second hypothesis. Moreover, changes in coupling ratio were usually related to a decrease in upper limb frequency when the upper limbs stepped at lower speeds than the lower limbs, but more rarely the other way around (Fig. 3b, 4). In the following, we will discuss the control mechanisms behind these results.

\section{1:1 Limb frequency relationship}

As stated, participants maintained a 1:1 frequency ratio between the upper and lower limbs by adjusting stance and participant in c displays an integer frequency ratio for all conditions; though, for the $1: 3 \mathrm{~km} / \mathrm{h}$ condition, a phase resetting from $1: 2$ to $1: 1$ occurs. These patterns (phase resetting and frequency drift) account for the frequency ratios that do not follow an integer pattern

swing times as also seen when adapting to upright bipedal walking on a split-belt treadmill (Reisman et al. 2005) (see Fig. 2). When the treadmill belts were moving at the same speed (Fig. 2, dashed lines), there was an expected decrease in cycle period and stance time, similar to that seen in the cat (Halbertsma 1983). However, an interesting result is that lower limb treadmill speed manipulations had no effect on stance and swing proportions in the upper limbs (Fig. 2e). Such a result may indicate that although upper limb absolute stance times are adjusted for changes in lower limb treadmill, the proportions of stance and swing are maintained and may not fully adapt to lower limb treadmill speed changes. The reason for this may be related to representations of limb movements in the motor cortex. In the intact cat, Zelenin et al. (2011) found that the vast majority of hindlimb neurons in motor cortex were also sensitive to changes in forelimb 
movements, in contrast with the forelimb motor cortical neurons which were sensitive to changes in hindlimb movements only in a small percentage. If a similar case is present in humans by which a greater percentage of lower limb neurons in the motor cortex are also sensitive to changes in upper limb movements, one may hypothesize that this mechanism could lead to the lack of change in upper limb stance and swing proportions.

If we extend the current study to bipedal human locomotion, the findings tend to support the hypothesis that upper limb swing during walking is, in some part, neural in origin (Dietz et al. 2001; Zehr et al. 2009). As previously stated, it is thought that arm swing during locomotion is governed by CPGs as hypothesized for lower limb motion. The tight coupling between cervical and lumbosacral CPGs that are shown here during crawling may result in the integer frequency ratios between the upper and lower limb movement in upright walking.

Deviations from a 1:1 limb coupling ratio: non-integer coupling ratios

When a 1:1 frequency relationship is no longer maintained between the upper and lower limbs, an integer ratio (1:2 or 1:3) is seen in most participants (see Fig. 4). This result corresponds to the integer relationship seen during swimming in humans (Wannier et al. 2001). Wannier et al. (2001) suggested that this is evidence of coordinated upper and lower limb CPGs. There were some participants in the current study who deviated from this pattern (e.g., the participant illustrated in Fig. 4a). Upon further inspection, it appears that these participants displayed greater inter-stride variance (up to 5 times) in gait characteristics (i.e., cycle period, stance time, etc.) than participants who followed an integer limb frequency relationship. Wannier et al. (2001) stated that trials with high variability were discarded in their analysis because it was suggested that the participant did not appropriately adapt to the task. Using IPL, we were able to identify that these particular participants displayed either a phase resetting or a frequency drift in the coordination of the upper and lower limbs. Therefore, it is also possible that the participants in the current study who did not display an integer frequency relationship had not adapted fully to that specific crawling condition.

Deviations from a 1:1 limb coupling ratio: biomechanical considerations

Coordination of adult crawling may be influenced by biomechanical factors. The current protocol required participants to crawl on their hands-and-feet with the purpose of making comparisons to quadrupedal locomotion. Human infants typically crawl on their hands-and-knees; however, hand-and-foot crawling is occasionally observed (Patrick et al. 2012). If the current protocol was used in adults who crawled on hands-and-knees, this would lead to a decrease in the functional length in the lower limbs. There is a possibility that this smaller functional length may affect interlimb coordination patterns, but this was not a particular question in the current study and can be addressed in future research. Moreover, the upper limbs are shorter than the lower limbs, and this may create a tendency for the upper limbs to cycle at a higher frequency. However, contrary to this prediction, the opposite was more likely to occur, and the upper limbs usually displayed lower frequencies (as seen in the proportion of participants displaying each pattern, see Fig. 3). Some quadrupedal animals (i.e., lemur) also have disproportionally longer lower limbs but still preserve a 1:1 limb coupling ratio. Furthermore, scapular retraction tends to be scaled to forelimb limb length in quadrupeds, therefore compensating for this lower functional range (Fischer and Blickhan 2006). A similar mechanism may be present during human crawling which would minimize this difference in limb length. Therefore, although biomechanical factors may influence limb motion, the fact that an integer ratio is maintained between the limbs (Fig. 4) suggests a neural origin for this pattern (Wannier et al. 2001; MacLellan et al. 2012a).

Deviations from a 1:1 limb coupling ratio: neural mechanisms

Now, one may ask why there is a preference for deviations from a 1:1 relationship to occur when the upper limb treadmill speed is decreased? Akay et al. (2006) reported that limb coupling ratio changes in decerebrate cats only occurred when lower limb treadmill speed decreased. The quite different result in the current study and those shown during swimming by Wannier et al. (2001) may be related to a possible overriding of upper limb CPG control by cortical projections. In the human, the upper limb is used for many non-rhythmic tasks when compared to the cat (i.e., reaching). In addition, Nakajima et al. (2000) compared different species of animals to show a positive correlation between advanced hand function and the development of direct cortical-motoneuronal projections onto hand muscles as well as a negative correlation between advanced hand function and propriospinal transmission. These patterns were thought to be related to the cortical system replacing the propriospinal system for fine hand movements. Therefore, this strong cortical influence on the upper limb in the human may facilitate a lowering of upper limb frequency at decreased upper limb treadmill speeds.

Alternatively (or possibly complementary to the above paragraph), another explanation for the preference of the upper limbs to cycle at a lower frequency in the human 
could be due to differing proportions of ascending and descending propriospinal neurons in the human. In the human, the major locus of CPG circuitry is in the lumbosacral enlargement and coupling of cervical and lumbosacral CPGs may depend on the strength of propriospinal connections. Indeed, cervical and lumbosacral enlargements in humans appear to be coupled by long propriospinal neurons projecting to different pools of alpha motor neurons across several spinal segments (Nathan et al. 1996). Moreover, reflex studies have suggested that stimulation of upper limb nerves can influence lower limb muscle activation during rhythmic activity and vice versa (Haridas and Zehr 2003; Zehr et al. 2004). Interestingly, Dietz et al. (2001) observed small or absent upper limb reflex responses during tibial nerve stimulation when participants were standing or sitting, suggesting task dependency of such responses. In contrast, in vitro studies on isolated neonatal rat spinal cords have suggested that lumbar CPGs generated a greater influence on cervical CPGs (Juvin et al. 2012; Juvin et al. 2005; Ballion et al. 2001). However, the differences in conclusions between these in vitro studies and the suggestions made in the current study may be due to the preparation (in vitro spinal cord versus intact human) being studied, development (neonatal versus adult), or a task dependence, as suggested by Thibaudier and Hurteau (2012). In any case, the findings in the current study lead us to speculate that the strength of these ascending propriospinal connections may be weaker in humans and ultimately allow cortical projections to have an influence on the cycling frequency of the upper limbs.

\section{Conclusions}

In conclusion, healthy adults generally preserve a $1: 1$ frequency relationship when hands-and-feet crawling on treadmills moving at differing speeds. This is accomplished through the manipulation of stance and swing times, with relative stance durations in the upper limbs only being affected by changes of the upper limb treadmill speed. This suggests that relative proportions of stance and swing do not adapt to changes in lower limb treadmill speeds (Fig. 2). When a 1:1 relationship is no longer maintained, an integer frequency ratio is present between the upper and lower limbs, supporting evidence of coupled CPG control. In addition, the upper limbs were more likely to display decreased frequencies when compared to the lower limbs (contrary to that seen previously in the cat) and may be due to weaker lumbosacral propriospinal projections to the cervical enlargement or a greater influence of cortical control on the human upper limbs.

Acknowledgments This work was supported by the Italian Ministry of Health, Italian Ministry of University and Research (PRIN grant),
Italian Space Agency (DCMC and CRUSOE grants) and European Union FP7-ICT program (AMARSi grant \#248311). The authors also thank Dr. G. Bosco for the comments on the preliminary results.

\section{References}

Akay T, McVea DA, Tachibana A, Pearson KG (2006) Coordination of fore and hind leg stepping in cats on a transversely-split treadmill. Exp Brain Res 175(2):211-222

Ballion B, Morin D, Viala D (2001) Forelimb locomotor generators and quadrupedal locomotion in the neonatal rat. Eur J Neurosci 14(10):1727-1738

de Sèze M, Falgairolle M, Viel S, Assaiante C, Cazalets JR (2008) Sequential activation of axial muscles during different forms of rhythmic behavior in man. Exp Brain Res 185:237-247

Dietz V, Michel J (2009) Human bipeds use quadrupedal coordination during locomotion. Ann NY Acad Sci 1164:97-103

Dietz V, Fouad K, Bastiaanse CM (2001) Neuronal coordination of arm and leg movements during human locomotion. Eur J Neurosci 14(11):1906-1914

Donker SF, Mulder T, Nienhuis B, Duysens J (2002) Adaptations in arm movements for added mass to wrist or ankle during walking. Exp Brain Res 146(1):26-31

Falgairolle M, de Sèze M, Juvin L, Morin D, Cazalets JR (2006) Coordinated network functioning in the spinal cord: an evolutionary perspective. J Physiol (Paris) 100:304-316

Fischer MS, Blickhan R (2006) The tri-segmented limbs of therian mammals: kinematics, dynamics, and self-stabilization-a review. J Exp Zool A Comp Exp Biol 305(11):935-952

Getchell N, Forrester L, Whitall J (2001) Individual differences and similarities in the stability, timing consistency, and natural frequency of rhythmic coordinated actions. Res Q Exerc Sport $72: 13-21$

Halbertsma JM (1983) The stride cycle of the cat: the modelling of locomotion by computerized analysis of automatic recordings. Acta Physiol Scand Suppl 521:1-75

Haridas C, Zehr EP (2003) Coordinated interlimb compensatory responses to electrical stimulation of cutaneous nerves in the hand and foot during walking. J Neurophysiol 90(5):2850-2861

Ivanenko YP, Cappellini G, Dominici N, Poppele RE, Lacquaniti F (2005) Coordination of locomotion with voluntary movements in humans. J Neurosci 25:7238-7253

Juvin L, Simmers J, Morin D (2005) Propriospinal circuitry underlying interlimb coordination in mammalian quadrupedal locomotion. J Neurosci 25(25):6025-6035

Juvin L, Le Gal JP, Simmers J, Morin D (2012) Cervicolumbar coordination in mammalian quadrupedal locomotion: role of spinal thoracic circuitry and limb sensory inputs. J Neurosci 32(3):953-965

MacLellan MJ, Qaderdan K, Koehestanie P, Duysens J, McFadyen BJ (2012a) Arm movements during split-belt walking reveal predominant patterns of interlimb coupling. Hum Mov Sci. doi:10.1016/j.humov.2012.08.001

MacLellan MJ, Ivanenko YP, Cappellini G, Sylos Labini F, Lacquaniti F (2012b) Features of hand-foot crawling behavior in human adults. J Neurophysiol 107(1):114-125

Nakajima K, Maier MA, Kirkwood PA, Lemon RN (2000) Striking differences in transmission of corticospinal excitation to upper limb motoneurons in two primate species. J Neurophysiol 84(2):698-709

Nathan PW, Smith M, Deacon P (1996) Vestibulospinal, reticulospinal and descending propriospinal nerve fibres in man. Brain 119:1809-1833

Orlovsky GN, Deliagina TG, Grillner S (1999) Neuronal control of locomotion. Oxford University Press, Oxford 
Patrick SK, Noah JA, Yang JF (2009) Interlimb coordination in human crawling reveals similarities in development and neural control with quadrupeds. J Neurophysiol 101:603-613

Patrick SK, Noah JA, Yang JF (2012) Developmental constraints of quadrupedal coordination across crawling styles in human infants. J Neurophysiol 107(11):3050-3061

Reisman DS, Block HJ, Bastian AJ (2005) Interlimb coordination during locomotion: what can be adapted and stored? J Neurophysiol 94(4):2403-2415

Sparrow WA, Newell KM (1994) The coordination and control of human creeping with increases in speed. Behav Brain Res 63(2):151-158

Tan U (2010) Uner Tan syndrome: history, clinical evaluations, genetics, and the dynamics of human quadrupedalism. Open Neurol $\mathbf{J}$ 4:78-89

Thibaudier Y, Hurteau MF (2012) Sensory regulation of quadrupedal locomotion: a top-down or bottom-up control system? J Neurophysiol 108(3):709-711
Wannier T, Bastiaanse C, Colombo G, Dietz V (2001) Arm to leg coordination in humans during walking, creeping and swimming activities. Exp Brain Res 141(3):375-379

Webb D, Tuttle RH, Baksh M (1994) Pendular activity of human upper limbs during slow and normal walking. Am J Phys Anthropol 93(4):477-489

Zehr EP, Frigon A, Hoogenboom N, Collins DF (2004) Facilitation of soleus H-reflex amplitude evoked by cutaneous nerve stimulation at the wrist is not suppressed by rhythmic arm movement. Exp Brain Res 159(3):382-388

Zehr EP, Hundza SR, Vasudevan EV (2009) The quadrupedal nature of human bipedal locomotion. Exerc Sport Sci Rev 37:102-108

Zelenin PV, Deliagina TG, Orlovsky GN, Karayannidou A, Dasgupta NM, Sirota MG, Beloozerova IN (2011) Contribution of different limb controllers to modulation of motor cortex neurons during locomotion. J Neurosci 31(12):4636-4649 